\title{
Sensing and Communication Energy Consumption in Static Sensor Network
}

\author{
G.N. Purohit \\ Department of Mathematics \\ AIM \& ACT, Banasthali University \\ Banasthali-304022
}

\author{
Seema Verma \\ Department of Electronics \\ AIM \& ACT, Banasthali University \\ Banasthali-304022
}

\author{
Megha Sharma \\ Department of Computer Science \\ AIM \& ACT, Banasthali University \\ Banasthali-304022
}

\begin{abstract}
A sensor network normally constitutes a Wireless ad-hoc network, meaning that in it each sensor supports a multi-hop routing algorithm (several nodes may forward data packets to the base station). But the sensor nodes suffer from constrained resources i.e., sensors have limited energy resources and their functionality continue only until their energy is drained out. Therefore the limited energy resource in these nodes demands an efficient consumption of these resources which should be managed wisely to extend the lifetime of sensors. In this paper, we study the problem of energy-efficient coverage and we propose various models for placing nodes in a static network. We consider a hexagonal model in two-dimensional deployment and a cubical/hexagonal-prism model for deploying sensors in space. Further, we have considered a rectangular grid divided into regular hexagons and the space covered by cubes and hexagonal-prisms. We have calculated the sensing energy and communication energy consumptions in each model and compared the same with the existing models. However, as far as we know there is no such model for covering the space.
\end{abstract}

\section{General Terms}

Sensing energy efficiency, Communication energy efficiency, Coverage et. al..

\section{Keywords}

energy-consumption, hexagonal-prism, cube, coverage models, sensing nodes.

\section{INTRODUCTION}

Wireless Sensor Networks are a new class of distributed systems that are an integral part of the physical space they inhibit [1]. A sensor network is an infrastructure comprised of sensing (measuring), computing, and communicating elements that give the ability to observe, and react to events and phenomena in specified environment. It is a fast growing and exciting research area that has attracted considerable attention in the recent past. With its origin in the early nineties, the subject of wireless sensor networks has seen an explosive growth in interest in both academia and industry [5].Unlike most computers which work primarily with data created by humans, sensor networks reason about the state of the worlds that embodies them [8]. Wireless sensor networks are providing tremendous benefit for a number of industries. The ability to add remote sensing points, without the cost of running wires, results in numerous benefits including energy and material savings, process improvements, labor savings, and productivity increases. Today Wireless Sensors Networks are being widely deployed.
A sensor network consists of multiple detection stations called sensor nodes, each of which is small, lightweight and portable. Every sensor node is equipped with a transducer, microcomputer, transceiver and power source. The transducer generates electrical signals based on sensed physical effects and phenomena. The microcomputer processes and stores the sensor output. The transceiver, which can be hard-wired or wireless, receives commands from a central computer and transmits data to that computer. The sensor nodes consume power for sensing, communicating and data processing. This power is derived from the electric utility or from a battery [4]. A sensor node might vary in size from that of a shoebox down to the size of a grain of dust. The cost of sensor nodes is similarly variable, ranging from hundreds of dollars to a few pennies, depending on the complexity of the individual sensor nodes [2]. As wireless sensor nodes are typical electronic devises, they can be equipped with a limited power source of less than 0.5-2 ampere-hour and 1.2-3.7 volts. Size and cost constraints on sensor nodes result in corresponding constraints on resources such as energy, memory, computational speed and communications bandwidth [2].

Energy is a primary constraint in the design of sensor networks. This fundamental energy constraint further limits everything from data sensing rates and link bandwidth, to node size and weight. Large volumes of sensor data generated will make the data transmission within the network to a single information sink with minimal latency and energy is a very challenging task [6]. This means, energy consumption is one of the most important performance metrics for wireless ad hoc sensor networks because it directly relates to the operational lifetime of the network [3]. As, ssensors have limited energy resources and their functionality continues until their energy drains, therefore, energy resources for sensor networks should be managed wisely to extend the lifetime of sensors [7]. A sensor node should be small in size, consume extremely low energy, operate in high volumetric densities, be autonomous and operate unattended, and be adaptive to the environment

In this paper, we have estimated energy efficiency in twodimensional and three-dimensional regions considering coverage by sensors of two different sensing ranges (i.e. Heterogeneous sensors). The region of interest is divided into regular hexagons in case of a plane region and the region of interest in space is divided into cubes. We have also considered a region consisting of a single hexagonal-prism. The sensor nodes are deployed on the vertices of these configurations. The sensing range of neighboring nodes are considered tangential to each other. To cover the uncovered area/region of these configurations we have deployed an additional sensor at the 
center of the configuration. This extra sensor has an adjustable sensing range. By adjustable sensing range we mean that the sensing range of the extra sensor is determined according to the gap to be covered by the extra sensor, which has not been covered by the sensors at the vertices.

Network Connectivity (for communication among sensors) is ensured by assuming that all the active sensor nodes form a minimum spanning tree (MST) and each sensor node adjusts its communication range to reach its farthest neighbor on the tree. As we know that the amount of energy consumed for communication by sensors depends upon the distance between the communicating sensors, therefore for the hexagon we assume that the energy consumed for communication by a sensor is proportional to the square of the distance from itself to its farthest neighbor on the tree by a factor of $\beta$ (power consumption per unit). For the cube and Hexagonal-Prism, energy consumed for communication by a sensor is proportional to cube of the distance from itself to its farthest neighbor on the tree by a factor of $\delta$ (power consumption per unit time). The model with the least sensing and communication energy consumption is considered to be the best in terms of sensing and communication energy efficiency respectively. We have considered the energy-efficiency of sensor network for these models. In the two-dimensional region we provide coverage using hexagons as tiles in the rectangular area and in the threedimensional space coverage is provided using cubes.

The remaining of the paper is organized as follows. Section 2 describes the Proposed Coverage Models, and provides an estimate of the sensing energy and communication energy consumption for these models. Section 3 provides an analysis of the energy consumptions for various models and Section 4 concludes the paper.

\section{PROPOSED COVERAGE MODELS}

In the two-dimensional area we consider a unit as a regular hexagon. Sensor nodes of two different strengths are used for deployment. Six nodes of equal strength are placed at the six vertices of the hexagon. The sensing radius is considered as half of the length of a side of hexagon. Still some area of the hexagon remains uncovered. For this another node of higher strength is placed at the center of the hexagon. . These nodes are placed in such a manner so that there is minimum overlapping. For deployment in space we consider two different types of regular solids, (i) a cube and (ii) a hexagonal-prism. Nodes of lower strength are placed at the vertices and a node of higher strength is placed at the center of the cube/hexagonal-prism. The side of a cube/hexagonal-prism is taken as twice the sensing range of the weaker node.

Next, we consider a rectangular region and divide it into regular hexagons. In case of rectangular parallelepiped, space is filled with regular cubes, and the nodes are deployed in a similar way, as in the case of unit models, cube/hexagonal-prism. We evaluate the sensing energy consumption and communication energy consumption for individual unit models and also for a two-dimensional area and a three-dimensional space. Larger is the area/space smaller is the per unit energy consumption (sensing and communication energy consumption) The detailed descriptions of sensing and communication energy consumption are included in the next section.

\section{SENSING ENERGY COMMUNICATION CONSUMPTION}

In this section, we consider and calculate the sensing energy and communication energy consumptions for different units (hexagonal-prism, cube, hexagon), and also for rectangular area and rectangular parallelepiped in space.

\subsection{Hexagonal-Prism}

(i) Sensing Energy Consumption

In the hexagonal-prism 12 sensing nodes are deployed at the 12 vertices, such that the sensing ranges of neighboring nodes are tangential. The height of the hexagonal-prism is taken equal to the length of the side of the regular hexagonal face and all vertical faces are squares of side equal to the side of hexagonalface. The sensing radius of the nodes at the vertices is $\mathrm{R}$, which is half of the length of the side of the hexagonal-face. The node placed at the center of the hexagonal-prism has sensing radius $2 \mathrm{R}$.

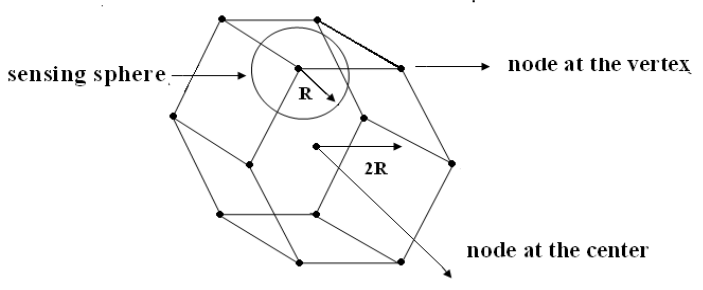

Figure 1: Hexagonal-Prism

In Fig. 1 we can see that the node at the vertex forms a sensing sphere. Similarly all other nodes deployed at the vertices and at the center of the hexagonal-prism forms sensing spheres.

Since the sensing range of a node is $\mathrm{R}$, each sensing node will cover a spherical volume $4 \pi R^{3} / 3$, which we call a sensing sphere for sensing. A part of this space will be inside the prism and the remaining will be outside the prism. We define the Coverage Density (CD), as the ratio of the total volume of the portions of the sensing region inside the hexagonal-prism (TV), divided by the volume of the hexagonal-prism (VM). Smaller the value of $\mathrm{CD}$, better the energy-efficiency.

The total volume of the portions of the sensing spheres inside the hexagonal prism, the volume of the hexagonal-prism and the Coverage Density of the hexagonal prism respectively are:

$$
\begin{gathered}
\mathrm{TV}=\frac{8}{3} \pi \mathrm{R}^{3}+\frac{32}{3} \pi \mathrm{R}^{3}=40 \pi \mathrm{R}^{3} / 3 \\
\mathrm{VM}=12 \sqrt{3} \mathrm{R}^{3}
\end{gathered}
$$




$$
\mathrm{CD}=\mathrm{TV} / \mathrm{VM}=40 \pi \mathrm{R}^{3} / 36 \sqrt{3} \mathrm{R}^{3}=2.014311
$$

Therefore, the sensing energy consumption of the sensors deployed in the hexagonal-prism is:

$$
\mathrm{SC}_{\text {hexagonat-prism }}=\mathrm{TV} \cdot \alpha=40 \pi \mathrm{R}^{3} \cdot \alpha / 3
$$

and, Sensing Energy Consumption per Unit Volume is:

$$
\begin{aligned}
& \mathrm{SEV}_{\text {hexagona-prism }}=\mathrm{SC}_{\text {hexagona-prism }} / \mathrm{VM} \\
& =40 \pi \mathrm{R}^{3} . \alpha / 36 \sqrt{3} \mathrm{R}^{3}=2.014311 \alpha
\end{aligned}
$$

where $\alpha$ is the power consumption per unit time.

\section{(ii) Communication Energy Consumption}

The minimum distance between any two adjacent sensing-nodes in the hexagonal-prism configuration is $\mathrm{d}_{\text {hexagonalprism }}=2 \mathrm{R}$. The consumption of energy in sensing by a sensing node is proportional to the sensing region inside the prism. The total sensing region $\left(\mathrm{P}_{\text {hexagonalprism }}\right)$ of all the 12 sensors at vertices and 1 sensor at the center is equivalent to sensing by $(2+1)=3$ sensors. Therefore, the Communication Energy Consumption (CC) and the Communication Energy Consumption per Unit Volume (CEV) for sensors deployed in the hexagonal-prism is:

$$
\begin{aligned}
& \mathrm{CC}_{\text {hexagonatprism }}=\mathrm{P}_{\text {hexagonatprism }} \cdot \delta \cdot \mathrm{d}^{3}{ }_{\text {hexagonatprism }} \\
& =(3) . \delta \cdot(2 \mathrm{R})^{3} \\
& \mathrm{CEV}_{\text {hexagonatprism }}=\mathrm{CC}_{\text {hexagonatprism }} / \mathrm{VM}= \\
& \text { (3). } \delta .(2 \mathrm{R})^{3} / 12 \sqrt{3} \mathrm{R}^{3}=1.154701 \delta
\end{aligned}
$$

where $\delta$ is the power consumption per unit.

\subsection{Cube}

\section{(i) Sensing Energy Consumption}

In case of a cube, eight sensing-nodes are deployed at the eight vertices. The sensing radius of the nodes at the vertices is $R$, which is half of the length of the side of the cube, and the radius of the sensing node placed at the center of the cube is $\sqrt{2} \mathrm{R}$. Since the sensing range of a node is $\mathrm{R}$, each sensing node will cover a spherical volume $4 \pi R^{3} / 3$, which we call a sensing sphere for sensing. A part of this space will be inside the cube and the remaining will be outside the cube.

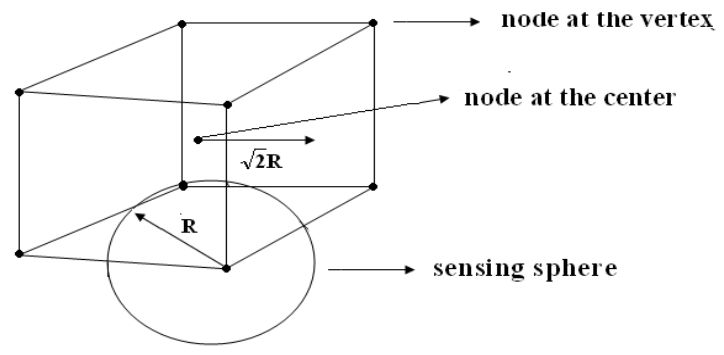

Figure 2: Cube

In Figure- 2 we can see that the node at the vertex forms a sensing sphere. Similarly all other nodes deployed at the vertices and at the center of the cube forms a sensing sphere.

The total volume of the portions of the sensing spheres inside the cube (TV), the volume of the cube (VM) and the Coverage Density $(\mathrm{CD})$ respectively are:

$$
\begin{aligned}
& \mathrm{TV}=\frac{4}{3} \pi \mathrm{R}^{3}+\frac{8 \sqrt{2}}{3} \pi \mathrm{R}^{3}=(4+8 \sqrt{2}) \pi \mathrm{R}^{3} / 3, \\
& \mathrm{VM}=8 \mathrm{R}^{3} \\
& \mathrm{CD}=\mathrm{TV} / \mathrm{VM}=(4+8 \sqrt{2}) \pi \mathrm{R}^{3} / 24 \mathrm{R}^{3} \\
& =2.003544
\end{aligned}
$$

So, the sensing energy consumption of sensors placed in the cube is:

$$
\mathrm{SC}_{\text {cube }}=\mathrm{TV} \cdot \alpha=(4+8 \sqrt{2}) \pi \mathrm{R}^{3} \cdot \alpha / 3
$$

and, Sensing Energy Consumption per Unit Volume is:

$$
\begin{aligned}
& \mathrm{SEV}_{\text {cube }}=\mathrm{SC}_{\text {cube }} / \mathrm{VM}= \\
& (4+8 \sqrt{2}) \pi \mathrm{R}^{3} \cdot \alpha / 24 \mathrm{R}^{3}=2.003544 . \alpha
\end{aligned}
$$

where $\alpha$ is the power consumption per unit.

For the deployment of cubes in space we consider a rectangular parallelepiped of length ' $\mathrm{L}$ ', breadth ' $\mathrm{B}$ ' and height ' $\mathrm{H}$ ', which is divided into cubes of sides of length $2 \mathrm{R}$. Sensors placed at the vertices of the cubes have uniform sensing range $R$, which is half of the length of the side of the cube. The sensing range of the sensor placed at the center of the cubes is $\sqrt{2} \mathrm{R}$. The entire space(volume) is covered by $m^{*} n * p$ cubes, where 


$$
\mathrm{L}=\mathrm{n} * 2 \mathrm{R}, \mathrm{B}=\mathrm{m} * 2 \mathrm{R} \text { and } \mathrm{H}=\mathrm{p} * 2 \mathrm{R}
$$

The number of cubes in a row are numbered 1 to $\mathrm{p}$. number of columns are numbered 1 to $\mathrm{n}$, and number of cubes along the height of the rectangular parallelepiped are numbered 1 to $\mathrm{m}$.

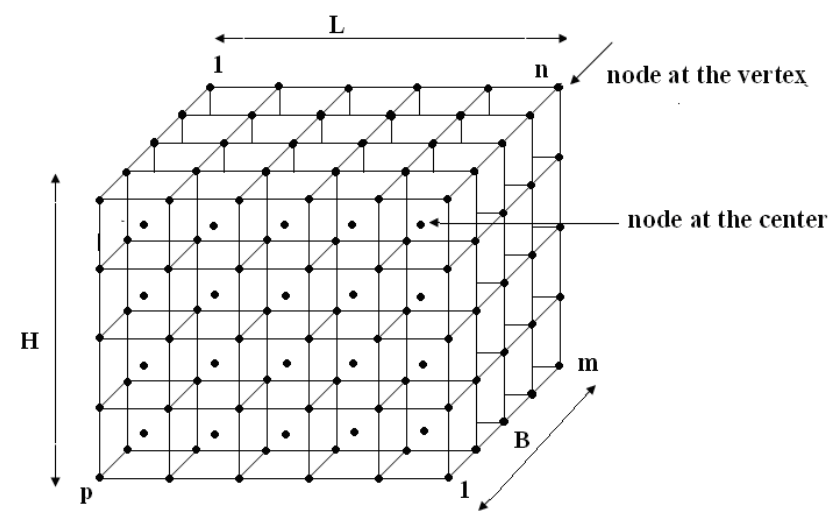

Figure 3: rectangular parallelepiped divided into cubes

The sensing energy consumptions for varying volumes are given in table 1:

Table 1. SEV when rectangular parallelepiped is divided into cubes

\begin{tabular}{|c|c|c|c|c|c|}
\hline \multicolumn{2}{|c|}{$\begin{array}{c}\text { Values of } \\
\text { m, n, p }\end{array}$} & $\begin{array}{c}\text { Volume of } \\
\text { parallelepip } \\
\text { ed }\end{array}$ & $\begin{array}{c}\text { Volume of } \\
\text { sensing } \\
\text { spheres in } \\
\text { parallelepipe } \\
\text { d }\end{array}$ & \\
\cline { 1 - 2 } $\mathbf{m}$ & $\mathbf{n}$ & $\mathbf{p}$ & $\begin{array}{c}\text { SEV } \\
\text { units) }\end{array}$ & & \\
\hline 3 & 4 & 4 & 196608 & 355328.3661 & $1.807293529 \alpha$ \\
\hline 4 & 4 & 5 & 327680 & 579352.5035 & $1.768043529 \alpha$ \\
\hline 4 & 5 & 6 & 491520 & 856167.3152 & $1.741876862 \alpha$ \\
\hline 5 & 6 & 7 & 860160 & 1466139.202 & $1.70449591 \alpha$ \\
\hline 6 & 6 & 7 & 1032192 & 1746505.602 & $1.692035592 \alpha$ \\
\hline
\end{tabular}

(ii) Communication Energy Consumption

The minimum distance between any two adjacent nodes in the cube is $d_{\text {cube }}=\sqrt{3} \mathrm{R}$. The consumption of energy in sensing by a sensing node is proportional to the sensing region inside the cube. The total sensing region $\left(\mathrm{P}_{\text {cube }}\right)$ of all the 8 sensors at vertices and 1 sensor at the center is equivalent to sensing by $(1+1)=2$ sensors.

Therefore, the Communication Energy Consumption (CC) and the Communication Energy Consumption per Unit Volume $(\mathrm{CEV})$ for the sensors placed on the cube is:

$$
\begin{aligned}
& \mathrm{CC}_{\text {cube }}=\mathrm{P}_{\text {cube }} \cdot \delta \cdot \mathrm{d}^{3} \text { cube }=(2) \cdot \delta \cdot(\sqrt{3} \mathrm{R})^{3} \\
& \mathrm{CEV}_{\text {cube }}=\mathrm{CC}_{\text {cube }} / \mathrm{VM} \\
& =(2) \cdot \delta \cdot(\sqrt{3} \mathrm{R})^{3} / 8 \mathrm{R}^{3}=1 \cdot 299038 \delta
\end{aligned}
$$

The corresponding communication energy consumptions by filling space with cubes for varying volumes are given in table 2:

Table 2. CEV when rectangular parallel pied is divided into cubes

\begin{tabular}{|c|c|c|c|c|c|}
\hline \multicolumn{2}{|c|}{$\begin{array}{c}\text { Values of } \\
\mathbf{m}, \mathbf{n , p}\end{array}$} & $\begin{array}{c}\text { Volume } \\
\text { of } \\
\text { parallel } \\
\text { epiped }\end{array}$ & $\begin{array}{c}\text { Volume of } \\
\text { sensing } \\
\text { spheres in } \\
\text { parallelepip } \\
\text { ed } \\
\text { m cubit } \\
\text { units) }\end{array}$ & $\mathbf{n}$ & \multicolumn{1}{|c|}{ CEV } \\
\hline 3 & 4 & 4 & 196608 & 355328.3661 & $1.055468461 \delta$ \\
\hline 4 & 4 & 5 & 327680 & 579352.5035 & $1.006754532 \delta$ \\
\hline 4 & 5 & 6 & 491520 & 856167.3152 & $0.974278579 \delta$ \\
\hline 5 & 6 & 7 & 860160 & 1466139.20 & $0.927884361 \delta$ \\
\hline 6 & 6 & 7 & 1032192 & 1746505.602 & $0.912419622 \delta$ \\
\hline
\end{tabular}

Fig. 4 below gives a graphical representation of the different values of sensing energy and communication energy consumptions per unit volume for the varying volumes when cubes are deployed in a rectangular parallelepiped. 


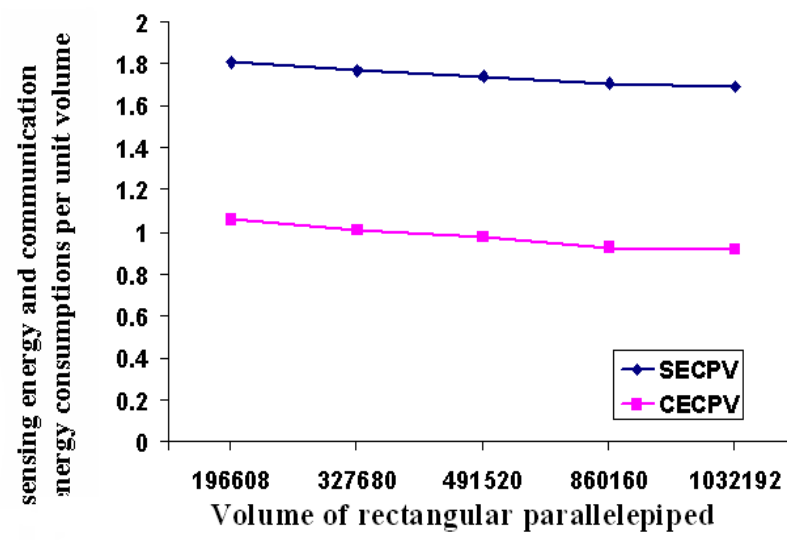

Figure 4: sensing and communication energy consumptions per unit volume for varying sizes of rectangular parallelepiped filled by cubes

To obtain the sensing energy and communication energy consumptions, the sensing range of the nodes at the vertices is taken as fixed, $\mathrm{R}=8 \mathrm{~m}$. The length $(\mathrm{L})$, breadth (B) and height $(\mathrm{H})$ are varied.

\subsection{Hexagon}

(i) Sensing Energy Consumption

In case of a hexagon there are six equal sensing nodes deployed at the six vertices of the hexagon, having sensing radius $R$, which is half of the length of the side of the hexagon, and the sensing range of the node placed at the center of the hexagon is a sensing disk of radius $\sqrt{3} \mathrm{R}$ Since the sensing range of a node is $\mathrm{R}$, each sensing node will cover a circular area $\pi \mathrm{R}^{2}$, which we call a sensing disk for sensing. A part of this space will be inside the hexagon and remaining will be outside the hexagon.

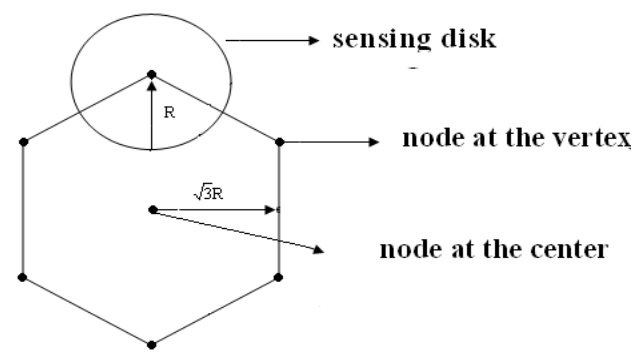

Figure 5: Hexagon
In Figure 5 we can see that the node at the vertex forms a sensing disk. Similarly all other nodes deployed at the vertices and at the center of the hexagon forms sensing disks.

The total area of the portions of the disks inside the hexagon (TA), area of the hexagon (AM), and the Coverage Density (CD) respectively are:

$$
\begin{gathered}
T A=2 \pi R^{2}+3 \pi R^{2}=5 \pi R^{2}, A M=6 \sqrt{3} R^{2} \\
C D=T A / V M=5 \pi R^{2} / 6 \sqrt{3} R^{2}=1.510733
\end{gathered}
$$

We suppose that the sensing energy consumption is proportional to the area of the sensing disk by a factor of $\lambda$, or the power consumption per unit. Then, sensing energy consumption for the hexagon is:

$$
\mathrm{SC}_{\text {hexagon }}=\mathrm{TA} \cdot \alpha=5 \pi \mathrm{R}^{2} \cdot \lambda
$$

and Sensing Energy Consumption per unit area is:

$$
\begin{aligned}
& \mathrm{SEA}_{\text {hexagon }}=\mathrm{SC}_{\text {hexagol }} / \mathrm{AM} \\
& =5 \pi \mathrm{R}^{2} \cdot \lambda \sqrt{3} \mathrm{R}^{2}=1.510733 . \lambda
\end{aligned}
$$

Now, we consider these parameters for a rectangular region in a plane that is a two-dimensional region. For this purpose we divide the whole region into regular hexagonal tiles. We consider a rectangular field of length ' $\mathrm{L}$ ' and breadth ' $\mathrm{B}$ ' which is divided into regular hexagons of sides of length $2 \mathrm{R}$. The sensors at the vertices of the hexagons have uniform sensing range $\mathrm{R}$, which is half of the length of the side of the hexagon, and the sensing range of all the sensors placed at the center of the hexagons is $\sqrt{3} R$. The whole rectangular area is covered by $\mathrm{m} * \mathrm{n}$ hexagons, where

$$
\begin{aligned}
& \mathrm{L}=(n-1) * 2 \mathrm{R} \sqrt{3} \\
& \text { and } \mathrm{B}=(2 m-1) * 2 \mathrm{R}
\end{aligned}
$$

However, the hexagons covering the boundary of the rectangle also cover some extra area. Nodes are not deployed on those vertices of these hexagons which are outside the rectangular region. Portions of sensing area of some of the nodes, deployed on the boundary of the rectangular area, lying outside the rectangular region is ignored, that is not included in sensing area.

The sketch of the model is given in Figure 6, where black dots presents the position of nodes 


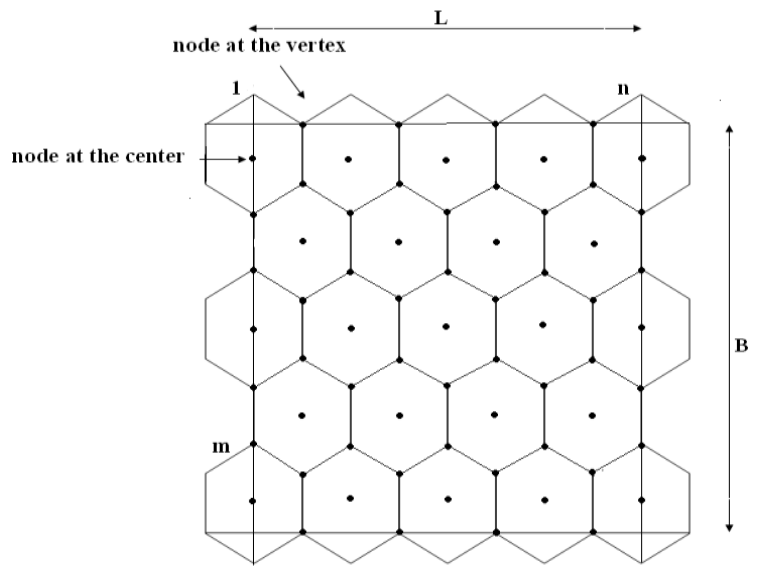

Figure 6: Hexagons deployed in a rectangular grid

The sensing energy consumptions for the varying rectangular areas are given in table 3 :

Table 3. SEA when a rectangular grid is divided into regular hexagons

\begin{tabular}{|c|c|c|c|c|}
\hline \multicolumn{2}{|c|}{$\begin{array}{c}\text { Values } \\
\text { of } \mathbf{~ m , ~}\end{array}$} & $\begin{array}{c}\text { Area of } \\
\text { rectangular } \\
\text { grid }\end{array}$ & $\begin{array}{c}\text { Area of } \\
\text { sensing disk }\end{array}$ & SEA \\
(square units) & n & & \\
\hline $\mathbf{m}$ & $\mathbf{n}$ & & 83398.4 & $1.27949853 \lambda$ \\
\hline 2 & 2 & 65180.53599 & 264262.4 & $1.208893 \lambda$ \\
\hline 3 & 3 & 218598.6683 & 601875.2 & $1.183429629 \lambda$ \\
\hline 4 & 4 & 508585.5427 & 1144467.2 & $1.170561306 \lambda$ \\
\hline 5 & 5 & 977708.0399 & & $1.162859082 \lambda$ \\
\hline 6 & 6 & 1668533.04 & 1940268.8 & \\
\hline
\end{tabular}

\section{(ii) Communication Energy Consumption}

The minimum distance between any two adjacent sensing-nodes in the hexagonal configuration is $\mathrm{d}_{\text {hexagon }}=2 \mathrm{R}$. The part of the sensor's communication energy used inside the hexagon is $\mathrm{P}_{\text {hexahon }}=(2+1)=3$.

Therefore, The Communication Energy Consumption (CC) and the Communication Energy Consumption per Unit Area (CEA) for the hexagon are:

$$
\begin{gathered}
\mathrm{CC}_{\text {hexagon }}=\mathrm{P}_{\text {hexahon }} \cdot \beta \cdot \mathrm{d}^{2} \text { hexagon }=(3) \cdot \beta \cdot(2 \mathrm{R})^{2} \\
\mathrm{CEA}_{\text {hexagon }}=\mathrm{CC}_{\text {hexagon }} / \mathrm{AM} \\
=(3) \cdot \beta \cdot(2 \mathrm{R})^{2} / 6 \sqrt{3} \mathrm{R}^{2}=1 \cdot 154701 \beta
\end{gathered}
$$

where $\beta$ is the power consumption per unit.

The communication energy consumptions for the varying rectangular areas are given in table 4:

Table 4. CEA when rectangular grid is divided into regular hexagons

\begin{tabular}{|l|l|c|c|c|}
\hline \multicolumn{2}{|c|}{$\begin{array}{c}\text { Values of } \\
\text { m, n }\end{array}$} & $\begin{array}{c}\text { Area of } \\
\text { rectangular grid } \\
\text { (square units) }\end{array}$ & $\begin{array}{c}\text { Area of } \\
\text { sensing disk }\end{array}$ & CEA \\
\hline m & n & & & \\
\hline 2 & 2 & 65180.53599 & 83398.4 & $0.977961 \beta$ \\
\hline 3 & 3 & 218598.6683 & 264262.4 & $0.923995 \beta$ \\
\hline 4 & 4 & 508585.5427 & 601875.2 & $0.904532 \beta$ \\
\hline 5 & 5 & 977708.0399 & 1144467.2 & $0.894697 \beta$ \\
\hline 6 & 6 & 1668533.04 & 1940268.8 & $0.888809 \beta$ \\
\hline
\end{tabular}

Fig. 7 below gives a graphical representation of the sensing energy and communication energy consumptions per unit area for varying area when a rectangular grid is divided into hexagonal tiles and nodes are placed in a manner already described.

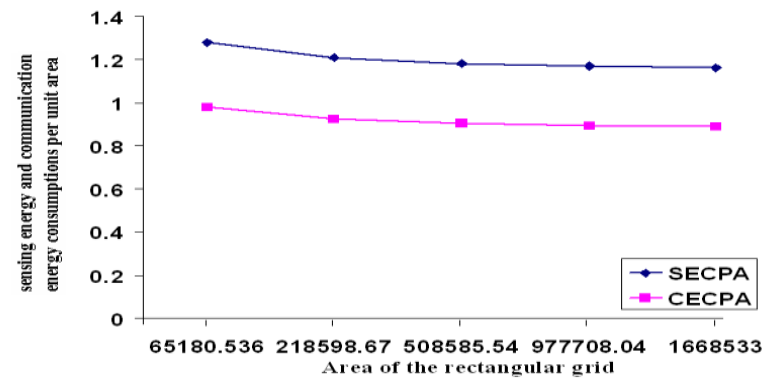

Figure 7: sensing and communication energy consumptions per unit area 
To obtain the sensing energy and communication energy consumptions, the sensing range of the nodes at the vertices is kept at a fixed value of $\mathrm{R}=8 \mathrm{~m}$. The length (L), and breadth (B) are varied.

\section{ANALYSIS OF THE PROPOSED MODELS}

A summary of the results for the sensing and communication energy consumption for the hexagon, cube and hexagonal-prism as unit models, and the hexagon and cube when deployed in two-dimensional area and three-dimensional space respectively are given in Table 5:

Table 5 . sensing and communication energy consumptions per unit area/volume for hexagon, cube, hexagonal-prism as unit models

\begin{tabular}{|l|c|c|c|}
\hline & Hexagon & Cube & $\begin{array}{c}\text { Hexagonal } \\
\text { Prism }\end{array}$ \\
\hline SEA/SEV & $1.512 \lambda$ & $2.004 \alpha$ & $2.014 \alpha$ \\
\hline CEA/CEV & $1.155 \beta$ & $1.299 \delta$ & $1.155 \delta$ \\
\hline
\end{tabular}

We can see here that Hexagon is best in terms of sensing energy and communication energy consumption per unit area/volume.However, communication energy efficiency remains unchanged in a hexagonal tile and hexagonalprism.Therefore, we can say that hexagonal model is best among

sensing and communication energy consumptions when a rectangular area is divided into regular hexagons are given in table 6

Table 6. SEA and CEA when rectangular grid is divided into rectangular hexagons. other models for the overall energy-effeciency (sensing and communication energy efficiency)

sensing and communication energy consumptions when space is filled by cubes is given in table 7 .

\begin{tabular}{|c|c|c|c|c|c|c|}
\hline \multicolumn{3}{|c|}{$\begin{array}{c}\text { Values } \\
\text { of } \mathbf{m}, \\
\mathbf{n}, \mathbf{p}\end{array}$} & \multirow{2}{*}{$\begin{array}{c}\begin{array}{c}\text { Volume } \\
\text { of } \\
\text { parallel }\end{array} \\
\text {-epiped } \\
\text { (cubit } \\
\text { units) }\end{array}$} & \multirow{2}{*}{$\begin{array}{c}\text { Volume of } \\
\text { sensing } \\
\text { spheres in } \\
\text { parallelepipe } \\
\text { d }\end{array}$} & \multirow[t]{2}{*}{ SEV } & \multirow[t]{2}{*}{ CEV } \\
\hline $\mathbf{m}$ & $n$ & p & & & & \\
\hline 3 & 4 & 4 & 196608 & 355328.3661 & $1.807293529 \alpha$ & $1.055468461 \delta$ \\
\hline 4 & 4 & 5 & 327680 & 579352.5035 & $1.768043529 \alpha$ & $1.006754532 \delta$ \\
\hline 4 & 5 & 6 & 491520 & 856167.3152 & $1.741876862 \alpha$ & $0.974278579 \delta$ \\
\hline 5 & 6 & 7 & 860160 & 1466139.202 & $1.70449591 \alpha$ & $0.927884361 \delta$ \\
\hline 6 & 6 & 7 & $\begin{array}{c}103219 \\
2\end{array}$ & 1746505.602 & $1.692035592 \alpha$ & $0.912419622 \delta$ \\
\hline
\end{tabular}

Table 7. SEV and CEV when rectangular parallelepiped is filled with cubes

We can see that the sensing/communication energy consumption per unit area/volume decreases as the area/volume in which sensors are deployed increases

\section{CONCLUSION}

In this paper, we have considered three models for deployment of sensors in a plane and in space i.e., hexagon, cube, hexagonal-prism, for testing the energy-efficiency (sensing and communication energy efficiency). In each model we have considered that neighboring sensing disks/spheres are meeting tangentially. Nodes are placed at the vertices of these configurations and the region inside the unit, not covered by the sensing disks/spheres placed at these vertices, is covered by sensing range of another node placed at the center of the unit. The node at center is of such a sensing range that it covers all the uncovered area/space. Analysis of the results reveals that the hexagon is the best in terms of sensing energy efficiency and the hexagon and hexagonal-prism are best in terms of communication energy efficiency. Overall we can say that hexagon is best in terms of energy-efficiency. 


\section{REFERENCES}

[1] Agarkar, S. A., Dr. Kulat, K.D., Dr. Kshirsagar, R.V. 2010. "WSN based Low Cost and Low Power EPM Design and Field Micro-Climate Analysis using Recent Embedded Controllers", International Journal of Computer Applications. Vol. 12, No.6

[2] Romer, K., Mattern, F. 2004. "The Design Space of Wireless Sensor Networks", IEEE Wireless Communications, pp. 54-61, Vol. 11, No. 6 Technical Report. University of Maryland at College Park.

[3] Gao, J. L. 2002. "Analysis of Energy Consumption for Ad Hoc Wireless Sensor Networks Using a Bit-Meter-per-Joule Metric", IPN Progress Report 42-150.

[4] Patel, N., Bhalekar, M., Nirav, P., Rizvi, S., Elleithy, K. "Reducing Power Consumption in Sensor Network Using Sensor MAC Protocol". Available on Google: http://www1bpt.bridgeport.edu/ elleithy/Conferences/C5_Redu cing Power Consumption in Sensor NetworkUsinSensorMac Protocol.pdf

[5] Ranganathan V. and Ponnusamy, V. 2009. "Energy Efficient Data Compression in Wireless Sensor Networks", The International Arab Journal of Information Technology. Vol. 6, No. 3.

[6] Arisha, K. A. , Youssef, M. A., Younis, M. F. "EnergyAware TDMA-Based MAC for Sensor Networks", Springer Link. Lecture Notes in Computer Science Ubiquitous Intelligence and Computing.

[7] Raghavendra, C. S. 2004. "Wireless sensor networks" Springer Verlag.

[8] Shukur, M. I., Chyan, L. S., Yap, V. V. .2009, "Wireless Sensor Networks:Delay Guarentee and Energy Efficient MAC Protocols" World Academy of Science, Engineering and Technology.

\section{AUTHORS PROFILE}

Prof. G. N. Purohit is a Professor in Department of Mathematics \& Statistics at Banasthali University (Rajasthan). Before joining Banasthali University, he was Professor and Head of the Department of Mathematics, University of Rajasthan, Jaipur. He had been Chief-editor of a journal.His present interest is in O.R., Discrete Mathematics and Communication networks. He has published around 40 research papers in various journals.

Dr. Seema Verma is an eminent researcher and working as Associate Professor in Department of Electronics, Banasthali University. She has completed her master's degree in Electronics in 1999 and got her Ph.D in 2003. Her areas of interest include Communication, Error Control Coding, TURBO CODES, OFDM, MIMO, MT-CDMA, Channel Modeling, VLSI design of communication systems etc. She is the investigator of the research projects given by UGC, DST \& AICTE. She is a research guide announced by UGC. She has many papers to her credit in many Reputed International Journals, National \& International Conferences. She is in the TPC of many International conferences and in the editorial board of many Research journals.

Megha Sharma received the B.C.A and M.C.A degree from I.G.N.O.U in 2004 and 2008, respectively. She is currently working towards a $\mathrm{Ph} . \mathrm{D}$ degree in computer Science at the Banasthali University of Rajasthan. Her research interests include wireless sensor networks with a focus on the coverage of wireless sensor networks. 